\title{
Worst Case to Average Case Reductions for Polynomials
}

\author{
Tali Kaufman* \\ MIT \& IAS \\ kaufmant@mit.edu
}

\author{
Shachar Lovett ${ }^{\dagger}$ \\ Weizmann Institute of Science \\ shachar.lovett@weizmann.ac.il
}

October 26, 2018

\begin{abstract}
A degree- $d$ polynomial $p$ in $n$ variables over a field $\mathbb{F}$ is equidistributed if it takes on each of its $|\mathbb{F}|$ values close to equally often, and biased otherwise. We say that $p$ has a low rank if it can be expressed as a bounded combination of polynomials of lower degree. Green and Tao GT07. have shown that bias imply low rank over large fields (i.e. for the case $d<|\mathbb{F}|$ ). They have also conjectured that bias imply low rank over general fields. In this work we affirmatively answer their conjecture. Using this result we obtain a general worst case to average case reductions for polynomials. That is, we show that a polynomial that can be approximated by few polynomials of bounded degree, can be computed by few polynomials of bounded degree. We derive some relations between our results to the construction of pseudorandom generators, and to the question of testing concise representations.
\end{abstract}

\footnotetext{
${ }^{*}$ Research supported in part by NSF Awards CCF-0514167 and NSF-0729011.

${ }^{\dagger}$ Research supported by ISF grant 1300/05
} 


\section{Introduction}

Let $\mathbb{F}$ be a prime finite field. Let $p: \mathbb{F}^{n} \rightarrow \mathbb{F}$ be a polynomial in $n$ variables over $\mathbb{F}$ of degree at most $d$. We say that $p$ is equidistributed if it takes on each of its $|\mathbb{F}|$ values close to equally often, and biased otherwise. We say that $p$ has a low rank if it can be expressed as a bounded combination of polynomials of lower degree, and high rank otherwise. More formally we consider the following definitions.

Definition 1 (bias). The bias of a function $f: \mathbb{F}^{n} \rightarrow \mathbb{F}$ is defined to be

$$
\operatorname{bias}(f)=\mathbb{E}_{X \in \mathbb{F}^{n}}\left[\omega^{f(X)}\right]
$$

where $\omega$ stands for the $|\mathbb{F}|$ root of unity, i.e. $\omega=e^{\frac{2 \pi}{|\mathbb{F}|}}$

Definition 2 (rank). Let $p(X)$ be a degree $d$ polynomial over $\mathbb{F}^{n} \cdot \operatorname{rank}_{d-1}(P)$ is the smallest integer $k$ such that there exist degree $d-1$ polynomials $q_{1}(X), \ldots, q_{k}(x)$, and a function $F: \mathbb{F}^{k} \rightarrow \mathbb{F}$, s.t. $p(X)=F\left(q_{1}(X), \ldots, q_{k}(X)\right)$.

Green and Tao [GT07] have shown that over large fields bias imply low rank.

Theorem 1 (Theorem 1.7 in GT07]). Let $p(X)$ be a degree d polynomial over $\mathbb{F}^{n}$, where $d<|\mathbb{F}|$. If $\operatorname{bias}(p) \geq \delta>0$, then $\operatorname{rank}_{d-1}(p) \leq c(\delta, d)$.

In their paper, Green and Tao conjecture that the restriction $d<|\mathbb{F}|$ can be removed, but their proof technique breaks down when $d \geq|\mathbb{F}|$. Note that over large fields things might behave differently than over small fields. One important example is the The Inverse Conjecture for the Gowers Norm. This conjecture roughly says that if the $d$-derivative of a polynomial is biased then that polynomial has a non-negligible correlation with some polynomial of degree $d-1$. The Inverse Conjecture for the Gowers Norm was proven to be true over large fields by GT07, but was proven to be false over small fields [GT07, LMS]. One of the main tools used for proving the conjecture over large fields was Theorem 1, that was proven over large fields.

One could ask what is the case with the above theorem, whether it remains true over smaller fields or it becomes false there. We show that the [GT07] result is true over general fields. In this respect, as opposed to the Inverse Conjecture for the Gowers Norm case, large and small fields behave similarly.

\subsection{Our Main Results}

Our first main theorem shows that bias imply low rank over general fields.

Theorem 2 (Bias imply low rank for general fields ). Let $p(X)$ be a degree d polynomial over $\mathbb{F}^{n}$, s.t. bias $(p) \geq \delta>0$. Then $\operatorname{rank}_{d-1}(p) \leq c(d, \delta)$. That is, there exist degree- $(d-1)$ polynomials $q_{1}(X), \ldots, q_{c}(x)$, and a function $F: \mathbb{F}^{c} \rightarrow \mathbb{F}$, s.t. $p(X)=F\left(q_{1}(X), \ldots, q_{c}(X)\right)$, and $c=c(d, \delta)$. Moreover, $q_{1}, \ldots, q_{c}$ are derivatives of the form $p(X+a)-p(X)$ where $a \in \mathbb{F}^{n}$.

Most of the technical part of the paper is dedicated to proving Theorem 2, The proof will go by induction on the degree $d$ of $p(X)$. Notice that for $d=1$ it holds trivially. So, we assume Theorem 2 to hold for all degrees smaller than $d$, and prove it for degree $d$. 
Our second main theorem is obtained as a corollary from Theorem 2, The theorem is a worst case to average case reduction for polynomials. It says that a polynomial that can be approximated by few polynomials of bounded degree, can be computed by few polynomials of bounded degree. We now move to define and prove this rigorously.

Definition 3 ( $\delta$-approximation). We say a function $f: \mathbb{F}^{n} \rightarrow \mathbb{F} \delta$-approximates $p(X)$ if:

$$
\left|\mathbb{E}_{X \in \mathbb{F}^{n}}\left[\omega^{p(X)-f(X)}\right]\right| \geq \delta
$$

Theorem 3 (Worst-case to average case reduction for polynomials of bounded degree). Let $p(X)$ be a polynomial of degree $d, g_{1}, \ldots, g_{c}$ polynomials of degree $k,(d, c, k=O(1))$ and $F: \mathbb{F}^{c} \rightarrow \mathbb{F}^{a}$ function s.t. the composition $G(x)=F\left(g_{1}(X), \ldots, g_{c}(X)\right) \delta$-approximates $p$. Then there exist $c^{\prime}$ polynomials $h_{1}, \ldots, h_{c^{\prime}}$ and a function $F^{\prime}: \mathbb{F}^{c^{\prime}} \rightarrow \mathbb{F}$ s.t.

$$
F^{\prime}\left(h_{1}(X), \ldots, h_{c^{\prime}}(X)\right) \equiv p(X)
$$

Moreover, $c^{\prime}=c^{\prime}(d, c, k)$ (i.e. independent of $n$ ) and each $h_{i}$ if of the form $p(X+a)-p(X)$ or $g_{j}(X+a)$ for $a \in \mathbb{F}^{n}$. In particular, if $k \leq d-1$ then also $\operatorname{deg}\left(h_{i}\right) \leq d-1$.

Proof. Develop $\omega^{F\left(z_{1}, \ldots, z_{c}\right)}: \mathbb{F}^{c} \rightarrow \mathbb{C}$ in the Fourier basis. If $F\left(g_{1}(X), \ldots, g_{c}(X)\right) \delta$-approximates $p(X)$, there must exist some Fourier coefficient which $\delta^{\prime}$-approximates $p\left(\delta^{\prime} \geq \delta|\mathbb{F}|^{-c}\right)$. That means, there exist $\alpha_{1}, \ldots, \alpha_{c} \in \mathbb{F}$ s.t. the polynomial

$$
p^{\prime}(x)=p(x)-\left(\alpha_{1} g_{1}(x)+\ldots \alpha_{c} g_{c}(x)\right)
$$

has bias at least $\delta^{\prime}$. Using Theorem 2 we get that there must exist at most $c^{\prime}$ derivatives of $p^{\prime}$ which computes it. We can now use them and $\alpha_{1} g_{1}+\ldots \alpha_{c} g_{c}$ to compute $p$.

\section{Significance of Results}

Bias imply low rank over general fields. Out first main theorem (Theorem 22) shows that over general fields there is a phenomena that bias imply low rank. Green and Tao [GT07] proved this for large fields. They left the case of small fields open. We answer their question affirmatively by showing that the "bias imply low rank" phenomena is robust and holds for all fields.

Worst case to average case reductions for polynomials. Our second main theorem (Theorem (3) shows that every polynomial, not necessarily biased, that is approximated by few other bounded degree polynomials, can be computed by few bounded degree polynomials. We view this result as a worst case to average case reduction for polynomials. I.e. in order to show that a polynomial can not be approximated by few bounded degree polynomials, it would be sufficient to show that the polynomial can not be calculated by few bounded degree polynomials. That later task might be easier. An example when such a scenario is relevant is the following. The papers GT07, LMS that disprove the Inverse Conjecture for the Gowers Norm needed to show that the symmetric polynomial $S_{4}$ over $\mathbb{F}_{2}$, i.e. $S_{4}\left(x_{1}, \ldots, x_{n}\right)=\sum_{i<j<k<l} x_{i} x_{j} x_{k} x_{l}$ can not be approximated by few degree 3 polynomials. Given the current result it could be sufficient (and maybe easier?) to show that $S_{4}$ can not be computed by few degree 3 polynomials. 
On the power of induction and relation to pseudorandom generators. Pseudorandom generator for polynomials of degree- $d$ is an efficient procedure that stretches $s$ field elements into $n \gg s$ field elements that can fool any polynomial of degree $d$ in $n$ variables. Pseudorandom generators are mostly interesting over small fields. One can use our first main theorem to provide an alternative proof to the correctness of the pseudorandom generators of [BV] that fool degree $d$ polynomials. The argument used by [BV] relied on the Gower Inverse Conjecture which turned out to be false for small fields [GT07, LMS. However, a better inspection of the [BV] argument shows that for proving the correctness of their pseudorandom generators one can only rely on the statement that "bias imply low rank" which we prove here.

The "bias imply low rank" idea suggests a robust way to construct pseudorandom generators for some complex function classes based on pseudorandom generators for simpler function classes. The last would be done by the following methodology. Either you are unbiased in which case you could fool whoever you wanted to fool, or you are a function of few functions of lower complexity, so by induction we obtain a construction of pseudorandom generator for functions of higher complexity classes (e.g. degree $d$ polynomials) given pseudorandom generators for functions of lower complexity classes (e.g. linear functions).

Relation to testing concise representations. Diakonikolas et al. [DLMORSW] suggest a general methodology to test whether a function on $n$ variables has a concise representation. The idea is to do testing by implicit learning. Their work provides property testers for several concise structures among them are $s$-sparse polynomials, size- $s$ algebraic circuits and more. Consider the following concise representation of degree $d$ polynomials. A polynomial of degree $d$ has a concise representation if it is a function of few polynomials of lower degree (i.e. if it has a low rank). We argue that one can use the "bias imply low rank" theorem in order to construct a tester that test for this concise representation. The tester first performs a low degree testing e.g. by [RS] to test that the given polynomial is of degree at most $d$, if the degree-tester rejects the tester rejects otherwise, the tester would approximate the bias of the polynomial. If the bias is large then by our first theorem the polynomial has low rank and the tester accepts, otherwise it rejects. The idea behind our approach for testing this concise representation of polynomials is robust in the following sense. It suggests a methodology for testing concise representation of some family (e.g depth $d$ circuits) given a membership tester for that family, and given that the family obeys the "bias imply low rank" principle. If these two conditions are met, one can construct a tester. The tester first test membership in the family and then estimate the bias. If the bias is high the rank is low and concise representation exists.

Extension to tensors Let $L(x, y)$ be a bilinear form over $\mathbb{F}^{n}$, i.e. a function of the form

$$
L(x, y)=x^{t} A y
$$

where $x, y \in \mathbb{F}^{n}$ and $A$ is a matrix. There is a close connection between the rank of the matrix and the bias of $L$. Dixon's Theorem ([MS]) tells us that the bias of $L$ (and in fact, all non-zero Fourier coefficients of $L$ ) has absolute value $c(\mathbb{F})^{-\operatorname{rank}\left(A+A^{t}\right)}$. The theory of higher dimensional multilinear forms, i.e. tensors, is much less understood. In particular, there is no single notion of tensor rank. We prove, as a direct corollary of Theorem 2 , that if we define the rank of a tensor as minimal number of lower degree multilinear forms needed to compute it, then bias imply low rank for tensors. 
Theorem 4. Let $L\left(X_{1}, \ldots, X_{d}\right)$ be a multilinear form of degree d s.t. bias $(L) \geq \delta>0$. Then, there exist degree- $(d-1)$ multilinear forms $q_{1}, \ldots, q_{c}$, each operating on $d-1$ variables out of $X_{1}, \ldots, X_{d}$, and a function $F: \mathbb{F}^{c} \rightarrow \mathbb{F}$, s.t.

$$
L\left(X_{1}, \ldots, X_{d}\right)=F\left(q_{1}\left(X_{1}, \ldots, X_{t_{1}-1}, X_{t_{1}+1}, \ldots, X_{d}\right), \ldots, q_{c}\left(X_{1}, \ldots, X_{t_{c}-1}, X_{t_{c}+1}, \ldots, X_{d}\right)\right)
$$

and $c=c(d, \delta)$. Moreover, $q_{1}, \ldots, q_{c}$ are derivatives of $L$.

Proof. We use Theorem 2 on $L$ as a degree $d$-polynomial, and observe that derivatives of $L$ are sums of $d$ degree- $(d-1)$ multilinear forms in $d-1$ variables of $X_{1}, \ldots, X_{d}$.

\subsection{Proof Overview}

The proof starts by a lemma of Bogdanov and Viola, showing that if a degree- $d$ polynomial $p(X)$ has bias, then we can build a constant-size circuit which approximates it, whose inputs are degree- $(d-1)$ polynomials (and in fact derivatives of $p$ ).

The technical heart of the paper is the proof of the following statement (Lemma 11): A biased polynomial of degree $d$ that is approximated by few degree $d-1$ polynomials can be computed by few degree $d-1$ polynomials.

In general our proof structure is similar in spirit to that of [GT07, however, there is a clear distinction between the two approaches that enable us to obtain the stronger result. The proof is by induction on the degree. An important notion in the proof is the following definition of a factor.

Definition 4 (Factor). A factor is a set of polynomials $g_{1}, \ldots, g_{m}: \mathbb{F}^{n} \rightarrow \mathbb{F}$. The number of polynomials in the set $m$ is the dimension of the factor. The maximum degree is the order of the factor. The polynomials of the factor divide the hyper-cube into $|\mathbb{F}|^{m}$ parts according to their joint image. Each such part is called a region.

All regions are of almost same size. The first step of the proof shows that given a set of polynomials of degree at most $d-1$ that approximates a degree- $d$ polynomial $p$, i.e. given a factor that approximates $p$, one can transform that factor into another factor of constant size that approximate $p$ in in which all of the regions are roughly of the same size. In order to obtain that we need the following definition of regularity.

Definition 5 (Regularity - informal). A factor is regular if the joint distribution of its polynomials is close to uniform. see formal definition in Definition 8 ,

The Regularity Lemma (see Lemma 5) shows that given a factor of constant dimension that approximate $p$, it can be transformed into another constant dimension factor which is regular and approximate $p$. Moreover, in a regular factor all regions are roughly of the same size (see Lemma 12).

Averaging arguments. Since we know that all regions are roughly of same size we can use averaging arguments to claim that most regions have large agreement with the polynomial $p$. These are denoted as almost good regions. The rest of the regions are denoted as bad regions but there are only few of them. We then show that almost good regions are good (i.e. they fully agree with $p$, see Lemma 13). We further show that $p$ must be fixed/constant over bad regions (Lemma 15). 
Hence we get that the polynomial $p$ is a function of the factor, and can be computed (and not only approximated) by the factor. So the heart of the proof is to show that almost good regions are good and that $p$ is constant on the few bad regions.

Almost good regions are good, and $p$ is fixed on the rest - a wishful scenario. For a set of variables $Y_{1}, Y_{2}, \ldots \in \mathbb{F}^{n}$ we denote by $Y_{I}=\sum_{i \in I} Y_{i}$. Since $p(x)$ is a polynomial of degree $d$ it satisfies cube constraints of the following form:

$$
p(x)=\sum_{I \subseteq[d+1],|I|>0}(-1)^{|I|+1} p\left(x+y_{I}\right)
$$

We show that for every point $x$ that belongs to an almost good region, there is a constraint that pass through it and all its other points (i.e. points of the form $x+y_{I}$ for $|I|>0$ ) belong to the good part of the same region. As all the values $p\left(x+y_{I}\right)=c$ for all good points in the region, we get that also $p(x)=c$. Hence we get that the value $p(x)$ is constant on the region for every $x$.

A question of interest here is given $x$ what is the probability that $x+y_{I}$ is in the region of $x$ for every $I$. Since the assignment of points to the region is determined by the values of the polynomials $g_{1}, \cdots, g_{m}$ that compose the factor, saying that for every $I, x+y_{I}$ is in the region of $x$ is equivalent to the following condition.

$$
\left[g_{i}(x)=g_{i}\left(x+y_{I}\right) \text { for all } i \in[m] \text { and } I \subseteq[d+1]\right]
$$

One can observe that for this condition to hold, due to the dependence between derivatives, it is sufficient to require the following.

$$
\left[g_{i}(x)=g_{i}\left(x+y_{I}\right) \text { for all } i \in[m] \text { and } I \subseteq[d+1] \text { s.t. } 1 \leq|I| \leq \operatorname{deg}\left(g_{i}\right)\right]
$$

Note that this condition by itself is not sufficient to ensure that $p(x)$ is assigned the value of the good part of the region. One need also to add the requirement that non of the $x+y_{I}$ fall in the bad part of the almost good region. Once making the calculations (Lemma 8) one can realize that if all the events that compose the above conditions were independent then $p(x)$ would have get the correct value. Hence we could have say that all the almost good regions are totally good (Lemma 13). So, $p$ agrees with the factor on all the regions but few. Given this, we show that $p$ is fixed also on the bad regions (Lemma 15) However, the above arguments work under a wishful assumption that all the considered events are independent. Much of the technical effort of this work goes into showing that the joint distribution of these events is close to being uniform, i.e. the events are almost independent.

Obtaining almost independence through interpolation. One way to prove the almostindependent argument is to relate the bias from independence to the bias of the $d$-derivatives of the $g_{i}$ 's that compose the factor. Using interpolation one can relate the bias of the $d$-derivative of the $g_{i}$ 's to the bias of the $g_{i}$ which should be small by the assumption about the regularity of the factor. The use of interpolation in the above argument is absolutely crucial, and that what allow [GT07] to get a result only for for the case $d<|\mathbb{F}|$. 
Our approach for dealing with general fields. In order to eliminate the need for interpolation that could hold only over large fields and in order to be able to claim that $p$ is indeed fixed on bad regions we define a stronger notion of regularity. The stronger regularity roughly requires uniformity of the $d$-derivatives of the polynomials in the factor

Definition 6 (Strong Regularity - informal). A factor is strongly regular if the joint distribution of the $d$-derivatives its polynomials is close to uniform. see formal definition in Definition 11 .

Based on this stronger regularity we prove counting lemmas (Lemmas 6 and 7) that enable us to get almost independence in the above sense without the need for interpolation. Thus, we obtain our results for general fields. In the following we discuss the usefulness of strong regularity. Using the strong regularity we allow all polynomial $g_{1}, \ldots, g_{m}$ to participate in the calculation of $g_{i}\left(x+Y_{I}\right)$, in contrast to the definition of Green and Tao which required only evaluations of the same $g_{i}$. This gives raise to the notion of an "independence degree" of a polynomial $\Delta\left(g_{i}\right)$ (which is between 1 and $\operatorname{deg}\left(g_{i}\right)$, and could be strictly lower than $\operatorname{deg}\left(g_{i}\right)$ given the other derivatives in the factor). Thus, instead of requiring a uniform joint distribution over the following set of events as Green and Tao do:

$$
\left[g_{i}(x)=g_{i}\left(x+y_{I}\right) \text { for all } i \in[m] \text { and } I \subseteq[d+1] \text { s.t. } 1 \leq|I| \leq \operatorname{deg}\left(g_{i}\right)\right]
$$

We could only required uniform joint distribution over a subset of the events, that is over:

$$
\left[g_{i}(x)=g_{i}\left(x+y_{I}\right) \text { for all } i \in[m] \text { and } I \subseteq[d+1] \text { s.t. } 1 \leq|I| \leq \Delta\left(g_{i}\right)\right]
$$

It turns out that this stronger notion of independence allows us to get almost independence between the variables, without the need of integration, which makes our result work for every field.

In the following we show an example that $\Delta\left(g_{i}\right)$ could be strictly smaller then $\operatorname{deg}\left(g_{i}\right)$.

Example 1. Consider the symmetric polynomial $S_{4}$ over $\mathbb{F}_{2}$, i.e.

$$
S_{4}\left(x_{1}, \ldots, x_{n}\right)=\sum_{i<j<k<l} x_{i} x_{j} x_{k} x_{l}
$$

Consider the fourth derivative of $S_{4}$, i.e. the polynomial in $X, Y_{1}, \ldots, Y_{4}$

$$
G\left(X, Y_{1}, \ldots, Y_{4}\right)=\sum_{I \subseteq[4]} S_{4}\left(X+Y_{I}\right)
$$

This polynomial corresponds to the 4-th Gowers Norm of $S_{4}$, and as was shown in [GT07] and [LMS, it has bias $1 / 8$. In particular, it cannot be independent (and so we would have defined $\Delta\left(S_{4}\right)$ to be at most 3$)$.

The strong regularity for polynomials that we define here might find some future applications.

\subsection{Organization}

The rest of the paper is organized as follows. We define required notation in Section 3 , We define and analyze regular and strongly regular factors in Section 4. We show that strong regularity implies almost independence in Section 5. We prove Theorem 2 in Section 6. 


\section{Preliminaries}

$\mathbb{F}$ if a fixed prime field. We work with constant degree polynomials over $\mathbb{F}^{n}$. We denote by capital letters $X, Y, \ldots$ variables in $\mathbb{F}^{n}$, and by small letters $x, y, a, \ldots$ values in $\mathbb{F}^{n}$. Degree of a polynomial will always mean total degree. Unless otherwise specified, when we speak of a degree $d$ polynomial, we mean in fact a polynomial of total degree at most $d$. For a set of variables $Y_{1}, Y_{2}, \ldots \in \mathbb{F}^{n}$ we denote by $Y_{I}=\sum_{i \in I} Y_{i}$, and similarly for a set of values $y_{1}, y_{2}, \ldots \in \mathbb{F}^{n}$. We write $u=v(1 \pm \epsilon)$ for $u \in[v(1-\epsilon), v(1+\epsilon)]$. When we speak of a growth function, we mean any monotone function $\mathcal{F}: \mathbb{N} \rightarrow \mathbb{N}$ (for example, $\mathcal{F}(n)=2^{n^{2}}$ ).

Definition 7 (Derivative space of a polynomial). For a polynomial $f(X)$, we define its derivative space to be the set

$$
\operatorname{Der}(f)=\left\{f(X+a)-f(X): a \in \mathbb{F}^{n}\right\}
$$

Notice that if $\operatorname{deg}(f)=k$ then all polynomials in $\operatorname{Der}(f)$ have degree at most $k-1$.

\section{Regularity of polynomials}

As we discussed in the introduction, the notion of regularity plays a major rule in our proof. Green and Tao in [GT07] suggested one notion of regularity (we refer to it henceforth as regularity) which limited their proof to work only for large fields (i.e. $d<|\mathbb{F}|$ ). We suggest a stronger notion of regularity (noted henceforth as strong regularity). This new notion of strong regularity is essential for obtaining a result for general fields. In the following we review the regularity definitions given by Green and Tao. Then, we present the notion of strong regularity and show that every constant factor that approximates a polynomial $p$ can be transformed into a constant factor that approximates $p$ and is also strongly regular. We end this section by showing that strong regularity implies almost independence for sets of variables that forms some specific structures. This almost independence is the crux of the proof of Theorem 2 ,

Definition 8 (Regularity of polynomials). Let $\mathcal{F}$ be any growth function. A set of polynomials $\left\{g_{1}, \ldots, g_{m}\right\}$ is called $\mathcal{F}$-regular if any linear combination $\alpha_{1} g_{1}(X)+\ldots \alpha_{m} g_{m}(X)$ cannot be expressed as a function of at most $\mathcal{F}(m)$ polynomials of degree $k-1$, where $k=\max \left\{\operatorname{deg}\left(g_{i}\right): \alpha_{i} \neq 0\right\}$ (i.e. $k$ is the maximal degree of $g_{i}$ appearing in the linear combination).

Green and Tao also define the notion of a refinement of a set of polynomials. Informally, a set $\left\{g_{1}, \ldots, g_{m}\right\}$ is a refinement of $\left\{f_{1}, \ldots, f_{s}\right\}$ if for any $i \in[s], f_{i}(x)$ can be computed given the values of $\left\{g_{1}(x), \ldots, g_{m}(x)\right\}$.

Definition 9 (Refinement). A set of polynomials $\left\{g_{1}, \ldots, g_{m}\right\}$ is a refinement of $\left\{f_{1}, \ldots, f_{s}\right\}$ if for any $i \in[s]$ there exists a function $F_{i}: \mathbb{F}^{m} \rightarrow \mathbb{F}$ s.t.

$$
f_{i}(X)=F_{i}\left(g_{1}(X), \ldots, g_{m}(X)\right)
$$

Green and Tao prove that for any growth function $\mathcal{F}$, any set of polynomials $F=\left\{f_{1}, \ldots, f_{s}\right\}$ can be refined to a $\mathcal{F}$-regular set $\left\{g_{1}, . ., g_{m}\right\}$, s.t. $m$ depends only on $s, \mathcal{F}$ and the maximal degree in $F$. Importantly, $m$ is independent of $n$. Green and Tao proof start by a set $\left\{f_{1}, \ldots, f_{s}\right\}$ which 
approximates $p(X)$ weakly, transform it to a set which approximates $p$ on almost all points, then use the regularity condition to show that it must in fact compute $p$ exactly. In order to prove this, they need to analyze the joint distribution of

$$
\left\{g_{i}\left(X+\sum_{t \in I} Y_{t}\right): i \in[m], I \subseteq[D]\right\}
$$

where $D=O(d), X, Y_{1}, \ldots, Y_{D} \in \mathbb{F}^{n}$ are independent variables. Lets denote by $Y_{I}=\sum_{i \in I} Y_{i}$. They prove that if we just look on the subset

$$
\left\{g_{i}\left(x+Y_{I}\right): i \in[m], I \subseteq[D],|I| \leq \operatorname{deg}\left(g_{i}\right)\right\}
$$

for any $x \in \mathbb{F}^{n}$, then these variables must be almost independent, and for any $|I|>\operatorname{deg}\left(g_{i}\right)$, $g_{i}\left(x+Y_{I}\right)$ is determined by $\left\{g_{i}\left(X+Y_{J}\right): J \subseteq I,|J| \leq \operatorname{deg}\left(g_{i}\right)\right\}$. Since the regularity requirement was for evaluations $\left\{g_{i}(X): i \in[m]\right\}$ they needed to use integration over $\mathbb{F}$ to get the independence result for evaluation on hypercubes, which limited their proof only to $d<\operatorname{char}(\mathbb{F})$.

We follow a similar approach, but in order to allow for $d \geq \operatorname{char}(\mathbb{F})$, we allow more freedom in the set of variables which are almost independent or fixed given the others. For a set of polynomials $g_{1}, \ldots, g_{m}$, we also have a "independence degree" $\Delta$ for every $g_{i}$ (which is between 1 and $\left.\operatorname{deg}\left(g_{i}\right)\right)$. Instead of requiring as Green and Tao do that:

$$
\left\{g_{i}\left(x+Y_{I}\right): i \in[m], I \subseteq[D],|I| \leq \operatorname{deg}\left(g_{j}\right)\right\}
$$

are almost independent, we demand only that

$$
\left\{g_{i}\left(x+Y_{I}\right): i \in[m], I \subseteq[D],|I| \leq \Delta\left(g_{j}\right)\right\}
$$

are almost independent. However, we also demand that for any $i \in[m]$ and $|I|>\Delta\left(g_{i}\right)$, the value of $g_{i}\left(x+Y_{I}\right)$ can be determined by $\left\{g_{j}\left(x+Y_{J}\right): J \subseteq I,|J| \leq \Delta\left(g_{j}\right)\right\}$. Notice that we allow all polynomial $g_{1}, \ldots, g_{m}$ to participate in the calculation of $g_{i}\left(x+Y_{I}\right)$, in contrast to the definition of Green and Tao which required only evaluations of the same $g_{i}$. It turns out that this stronger notion of independence allows us to get almost independence between the variables, without the need of integration, which makes our result work for every field.

We now move to formally define our notion of strong regularity, and to show it implies the almost independence/total dependence structure we have just described. We first extend the definition of a derivative space to several polynomials in several variable sets.

Definition 10 (Derivative space). For a set of polynomials $F=\left\{f_{1}(X), \ldots, f_{s}(X)\right\}$ we define:

$$
\operatorname{Der}(F)=\left\{f_{i}(X+a)-f_{i}(X): i \in[s], a \in \mathbb{F}^{n}\right\}
$$

Similarly, for a set of polynomials in several variables $F=\left\{f_{1}\left(Y_{1}, \ldots, Y_{k}\right), \ldots, f_{s}\left(Y_{1}, \ldots, Y_{k}\right)\right\}\left(Y_{1}, \ldots, Y_{k} \in\right.$ $\left.\mathbb{F}^{n}\right)$ we define:

$$
\operatorname{Der}(F)=\left\{f_{i}\left(Y_{1}+a_{1}, \ldots, Y_{k}+a_{k}\right)-f_{i}\left(Y_{1}, \ldots, Y_{k}\right): i \in[s], a_{1}, \ldots, a_{k} \in \mathbb{F}^{n}\right\}
$$

Notice that if the maximal degree of polynomials in $F$ is $k$, then the maximal degree of polynomials in $\operatorname{Der}(F)$ is at most $k-1$. We now define strong regularity. 
Definition 11 (Strong regularity of polynomials). Let $\mathcal{F}$ be any growth function. Let $G=$ $\left\{g_{1}, \ldots, g_{m}\right\}$ be a set of polynomials and $\Delta: G \rightarrow \mathbb{N}$ be a mapping from $G$ to the natural numbers. We say the set $G$ is strong $\mathcal{F}$-regular with the degree bound $\Delta$ if:

1. For any $i \in[m], 1 \leq \Delta\left(g_{i}\right) \leq \operatorname{deg}\left(g_{i}\right)$.

2. For any $i \in[m]$ and $r>\Delta\left(g_{i}\right)$, let $X$ and $Y_{1}, Y_{2}, \ldots, Y_{r}$ be variables in $\mathbb{F}^{n}$. There exist a function $F_{i, r}$ s.t.

$$
g_{i}\left(X+Y_{[r]}\right)=F_{i, r}\left(g_{j}\left(X+Y_{J}\right): j \in[m], J \subseteq[r],|J| \leq \Delta\left(g_{j}\right)\right)
$$

3. For any $r \geq 0$, let $X$ and $Y_{1}, \ldots, Y_{r}$ be variables in $\mathbb{F}^{n}$. Let $\left\{\alpha_{i, I}\right\}_{i \in[m], I \subseteq[r],|I| \leq \Delta\left(g_{i}\right)}$ be any collection of field elements, not all zero. Let $a\left(X, Y_{1}, \ldots, Y_{r}\right)$ stand for the linear combination:

$$
a\left(X, Y_{1}, \ldots, Y_{r}\right)=\sum_{i \in[m], I \subseteq[r],|I| \leq \Delta\left(g_{i}\right)} \alpha_{i, I} g_{i}\left(X+Y_{I}\right)
$$

Let $G^{\prime} \subseteq G$ be the set of all $g_{i}$ 's which appear in $a$, i.e.:

$$
G^{\prime}=\left\{g_{i} \in G: \exists I \alpha_{i, I} \neq 0\right\}
$$

There does not exist polynomials $h_{1}, \ldots, h_{l} \in \operatorname{Der}\left(G^{\prime}\right), l \leq \mathcal{F}(m)$ s.t. $a\left(X, Y_{1}, \ldots, Y_{r}\right)$ can be expressed as:

$$
H\left(h_{1}\left(X+Y_{I_{1}}\right), \ldots, h_{l}\left(X+Y_{I_{l}}\right)\right)
$$

for $I_{1}, \ldots, I_{l} \subseteq[r]$ and some function $H: \mathbb{F}^{l} \rightarrow \mathbb{F}$.

If the set $G$ satisfies only (1) and (2), we say $G$ is pre-strong-regular (notice that $\mathcal{F}$ appears only in $(3))$.

We first prove, similar to the proof in [GT07, that any set of polynomials can be refined to a strong $\mathcal{F}$-regular set, where the size of the resulting set depends only on the size of the original set, and the maximal degree of polynomials in it. Also, the refining set is contained in the space of iterated derivatives of the original polynomials.

We now formally define the space of iterated derivatives.

Definition 12 (Space of iterated derivatives). For a polynomial set $F$, we define its iterated derivative set $\operatorname{Der}_{C}$ to be the set of taking at most $C$ derivatives of $F$, i.e.

$$
\begin{gathered}
\operatorname{Der}_{0}(F)=F \\
\operatorname{Der}_{C}(F)=\operatorname{Der}\left(\operatorname{Der}_{C-1}(F)\right) \cup \operatorname{Der}_{C-1}(F)
\end{gathered}
$$

Lemma 5 (Strong-Regularity Lemma). Let $\mathcal{F}$ be any growth function. Let $F=\left\{f_{1}, \ldots, f_{s}\right\}$ be a set of polynomials of maximal degree $k$. There exist a refinement $G=\left\{g_{1}, \ldots, g_{m}\right\}$ of $F$ s.t.

1. The maximal degree of polynomials in $G$ is also at most $k$

2. The set $G$ is strong $\mathcal{F}$-regular. 
3. The size $m$ of $G$ is a function of only $\mathcal{F}, s$ and $k$. Importantly, it is independent of $n$.

4. There exists $C=C(\mathcal{F}, s, k)$ s.t. $G \subseteq \operatorname{Der}_{C}(F)$

Proof. We will start by defining a pre-strong-regular set $G$ from $F$, and will keep refining it until we reach a strong $\mathcal{F}$-regular set. Our set $G$ will also be in $\operatorname{Der}_{i}(F)$ at the $i$-th iteration. We will finish by showing that the refinement process must end in a finite number of steps.

We start by defining $\Delta: F \rightarrow \mathbb{N}$ by $\Delta\left(f_{i}\right)=\operatorname{deg}\left(f_{i}\right)$, and set the initial value of $G$ to be $F$. To show that the initial $G$ is pre-strong-regular with the degree bound $\Delta$, observe that for any $r>\operatorname{deg}\left(f_{i}\right)$, deriving $f_{i} r$-times yields the zero polynomial. Thus, if $Y_{1}, \ldots, Y_{r}$ are variables, we have the identity:

$$
f_{i}\left(X+Y_{[r]}\right)=\sum_{I \subsetneq[r]}(-1)^{r-|I|+1} f_{i}\left(X+Y_{I}\right)
$$

Since we can do this for any $r>\operatorname{deg}\left(f_{i}\right)$, we can continue and express $f_{i}\left(X+Y_{[r]}\right)$ as a linear combination of $\left\{f_{i}\left(X+Y_{I}\right): I \subseteq[r],|I| \leq \operatorname{deg}\left(f_{i}\right)\right\}$. Thus, $G$ is pre-strong-regular with the degree bound $\Delta$.

We will continue to refine $G$ as long as it is not strong $\mathcal{F}$-regular. Assume $G=\left\{g_{1}, \ldots, g_{m}\right\}$ at some iteration is not strong- $\mathcal{F}$-regular. By definition, there is some $r \geq 0$ and coefficients $\left\{\alpha_{i, I}\right\}_{i \in[m], I \subseteq[r],|I| \leq \Delta\left(g_{i}\right)}$ s.t. the linear combination:

$$
a\left(X, Y_{1}, \ldots, Y_{r}\right)=\sum_{i \in[m], I \subseteq[r],|I| \leq \Delta\left(g_{i}\right)} \alpha_{i, I} g_{i}\left(X+Y_{I}\right)
$$

can be expressed as a function of $l \leq \mathcal{F}(m)$ polynomials $h_{1}, \ldots, h_{l} \in \operatorname{Der}\left(G^{\prime}\right)$, where $G^{\prime}=\{i \in[m]$ : $\left.\exists I \alpha_{i, I} \neq 0\right\}$ is the set of all $g_{i}$ 's participating in the linear combination.

Let $g_{i_{0}}$ be a polynomial of maximal degree $k$ in $G^{\prime}$ and let $I_{0}$ be a maximal $I$ in respect to inclusion s.t. $\alpha_{i_{0}, I_{0}} \neq 0$. Notice that we must have that $\left|I_{0}\right| \leq \Delta\left(g_{i_{0}}\right)$. We have:

$$
\sum_{i \in[m], I \subseteq[r],|I| \leq \Delta\left(g_{i}\right)} \alpha_{i, I} g_{i}\left(X+Y_{I}\right)=H\left(h_{1}\left(X+Y_{J_{1}}\right), \ldots, h_{l}\left(X+Y_{J_{l}}\right)\right)
$$

for some function $H: \mathbb{F}^{l} \rightarrow \mathbb{F}$.

Notice first that $\operatorname{deg}\left(h_{i}\right) \leq k-1$ for all $i \in[l]$. Substitute in the expression $Y_{i}=0$ for all $i \notin I_{0}$. We get that $g_{i_{0}}\left(X+Y_{I_{0}}\right)$ can be expressed as a function of $\left\{g_{i_{0}}\left(X+Y_{J}\right): J \subsetneq I_{0}\right\}$, $\left\{g_{j}\left(X+Y_{J}\right): j \neq i, J \subseteq I_{0},|J| \leq \Delta\left(g_{j}\right)\right\}$ and $\left\{h_{j}\left(X+Y_{J}\right): J \subseteq I_{0},|J| \leq \operatorname{deg}\left(h_{j}\right)\right\}$. Thus, if we add the polynomials $h_{1}, \ldots, h_{l}$ to $G$ (and set $\left.\Delta\left(h_{i}\right)=\operatorname{deg}\left(h_{i}\right)\right)$, we can reduce $\Delta\left(g_{j}\right)$ to $\left|I_{0}\right|-1$. If we reduced it to zero, we can remove $g_{j}$ entirely from $G$. The resulting $G$ will be our set for the next iteration.

In order to prove that the refinement process ends after a finite number of iterations (depending on the initial size of $F$ and its maximal degree), notice that at each iteration, the sum of $\Delta\left(g_{i}\right)$ for all $g_{i} \in G$ with some degree $d^{\prime}$ reduces by at least 1 , where the new polynomials added are all of degree strictly smaller than $d^{\prime}$, and their number is bounded (as a function of $\mathcal{F}$ and the size of $G$ at the beginning of the iteration). So the total number of iterations is some Ackerman-like function of the initial number of polynomials, their maximal degree and the growth function $\mathcal{F}$. 


\section{Almost independence by strong regularity}

In the following we prove that strong regularity induces almost independence/total dependence structure over general sets of variables. The following lemmas are the main technical building blocks in the proof of Theorem 2 .

We start by proving a lemma correlating applications of $g_{i}$ on sums below the degree bound $\Delta$ to all sums over a set of variables.

Lemma 6. Let $G=\left\{g_{1}, \ldots, g_{m}\right\}$ be a strong-regular set with degree bound $\Delta$. Let $x, x^{\prime} \in \mathbb{F}^{n}$ be two points s.t. $g_{i}(x)=g_{i}\left(x^{\prime}\right)$ for all $i \in[m]$. Let $y_{1}^{\prime}, \ldots, y_{k}^{\prime} \in \mathbb{F}^{n}$ be values for some $k \geq 1$, and let $Y_{1}, \ldots, Y_{k} \in \mathbb{F}^{n}$ be $k$ random variables. Then the following two events are equivalent:

1. $A=\left[g_{i}\left(x+Y_{I}\right)=g_{i}\left(x^{\prime}+y_{I}^{\prime}\right)\right.$ for all $i \in[m]$ and $\left.I \subseteq[k]\right]$

2. $B=\left[g_{i}\left(x+Y_{I}\right)=g_{i}\left(x^{\prime}+y_{I}^{\prime}\right)\right.$ for all $i \in[m]$ and $I \subseteq[k]$ s.t. $\left.1 \leq|I| \leq \Delta\left(g_{i}\right)\right]$

Proof. It is obvious that if $A$ holds then also $B$ holds. Assume that $B$ holds, i.e. that

$$
g_{i}\left(x+Y_{I}\right)=g_{i}\left(x^{\prime}+y_{i}^{\prime}\right)
$$

for all $i \in[m]$ and $I \subseteq[k]$ s.t. $|I| \leq \Delta\left(g_{i}\right)$. Take some $I$ s.t. $I>\Delta\left(g_{i}\right)$. We need to show that also $g_{i}\left(x+Y_{I}\right)=g_{i}\left(x^{\prime}+y_{I}^{\prime}\right)$. Since $|I|>\Delta\left(g_{i}\right)$ we know by the strong regularity of $G$ that there is a function $F_{i, I}$ s.t.

$$
g_{i}\left(X+Y_{I}\right)=F_{i, I}\left(g_{j}\left(X+Y_{J}\right): j \in[m], J \subseteq I,|J| \leq \Delta\left(g_{j}\right)\right)
$$

By first substituting $X=x$ to compute $g\left(x+Y_{I}\right)$, and then substituting $X=x^{\prime}$ and $Y_{j}=y_{j}^{\prime}$ to compute $g\left(x^{\prime}+y_{I}^{\prime}\right)$, and using that both $g_{j}(x)=g_{j}\left(x^{\prime}\right)$ for all $j \in[m]$ and the assumption that $B$ holds, we get that also $g_{i}\left(x+Y_{I}\right)=g_{i}\left(x^{\prime}+y_{I}^{\prime}\right)$.

We now prove a lemma showing that points which are sum of at most $\Delta\left(g_{i}\right)$ points for all $g_{i}$ are simultaneously almost disjoint, provided that $\mathcal{F}$ is large enough. Remember that we are in the process of proving Theorem 2 for degree $d$ by induction. Thus, we assume it to hold for all degrees $d^{\prime}<d$, and in particular to all linear combinations of $g_{1}, \ldots, g_{m}$.

Lemma 7. Let $\gamma=\gamma(m)$ be an error term. Let $Y_{1}, \ldots, Y_{k} \in \mathbb{F}^{n}$ be random variables for some $k \geq 1$. Assume $\mathcal{F}$ is large enough (as a function of $\gamma$ and $k$ ). Assume $g_{1}, \ldots, g_{m}$ are strong $\mathcal{F}$-regular with degree bound $\Delta$. For any non-empty $I \subseteq[k]$ let $x_{I} \in \mathbb{F}^{n}$ be some point, and $a^{(I)}=\left(a_{1}^{(I)}, \ldots, a_{k}^{(I)}\right) \in \mathbb{F}^{k}$ s.t.

- $a_{i}^{(I)} \neq 0$ for all $i \in I$

- $a_{i}^{(I)}=0$ for all $i \notin I$

Then the joint distribution of

$$
\left(g_{i}\left(x_{I}+\sum_{i \in I} a_{i}^{(I)} Y_{i}\right): i \in[m], I \subseteq[k], 1 \leq|I| \leq \Delta\left(g_{i}\right)\right)
$$

is $\gamma$-close to the uniform distribution on $\mathbb{F}^{\sum_{i=1}^{m} \sum_{j=1}^{\Delta\left(g_{i}\right)}\left(\begin{array}{c}k \\ j\end{array}\right)}$. 
Before proving Lemma 7, we give an immediate corollary of it and Lemma 6;

Corollary 8. Let $x, x^{\prime} \in \mathbb{F}^{n}$ be two points s.t. $g_{i}(x)=g_{i}\left(x^{\prime}\right)$ for all $i \in[m]$. Let $y_{1}^{\prime}, \ldots, y_{k}^{\prime} \in \mathbb{F}^{n}$ be values for some $k \geq 1$, and let $Y_{1}, \ldots, Y_{k} \in \mathbb{F}^{n}$ be $k$ random variables. Then

$$
\mathbb{P}\left[g_{i}\left(x+Y_{I}\right)=g_{i}\left(x^{\prime}+y_{I}^{\prime}\right) \forall i \in[m], I \subseteq[k]\right]=|\mathbb{F}|^{-\sum_{i=1}^{m} \sum_{j=1}^{\Delta\left(g_{i}\right)}\left(\begin{array}{c}
k \\
j
\end{array}\right)}(1 \pm \gamma)
$$

We need the following simple lemma for the proof of Lemma 7 . It states that a random derivative of a biased polynomial is also biased.

Lemma 9. Let $h\left(Y_{1}, \ldots, Y_{k}\right)$ be a polynomial with bias $\delta$. Let $h^{\prime}$ be the derivation of $h$ in variables $Y_{1}, \ldots, Y_{r}$ by directions $Z_{1}, \ldots, Z_{r},(r \leq k)$ i.e.

$$
h^{\prime}\left(Y_{1}, \ldots, Y_{k}, Z_{1}, \ldots, Z_{r}\right)=\sum_{w \in\{0,1\}^{r}}(-1)^{|w|} h\left(Y_{1}+w_{1} Z_{1}, \ldots, Y_{r}+w_{r} Z_{r}, Y_{r+1}, \ldots, Y_{k}\right)
$$

where $|w|$ denotes the hamming weight of $w$. Then bias $\left(h^{\prime}\right) \geq \delta^{2^{r}}$.

Proof. We apply Cauchy-Schwartz. It's enough to prove for $k=2$ and $r=1$ because we can group variables.

$$
\begin{aligned}
\operatorname{bias}\left(h^{\prime}\right)= & \mathbb{E}_{Y_{1}, Y_{2}, Z_{1} \in \mathbb{F}^{n}}\left[\omega^{h\left(Y_{1}, Y_{2}\right)-h\left(Y_{1}+Z_{1}, Y_{2}\right)}\right]=\mathbb{E}_{Y_{2} \in \mathbb{F}^{n}}\left[\left(\mathbb{E}_{Y_{1} \in \mathbb{F}^{n}}\left[\omega^{h\left(Y_{1}, Y_{2}\right)}\right]\right)^{2}\right] \geq \\
& \left(\mathbb{E}_{Y_{1}, Y_{2} \in \mathbb{F}^{n}}\left[\omega^{h\left(Y_{1}, Y_{2}\right)}\right]\right)^{2}=\delta^{2}
\end{aligned}
$$

Proof. (of Lemma 7) We start by using the well known fact, that if a distribution over $\mathbb{F}^{r}$ is not uniform, it must have some biased functional. If the distribution we study is $\gamma$-far from uniform, then there must be a linear functional on $\left\{g_{i}\left(x_{I}+\sum_{i \in I} a_{i}^{(I)} Y_{i}\right): i \in[m], I \subset[k],|I| \leq \Delta\left(g_{i}\right)\right\}$ with some non-negligible bias depending on $\gamma$. We will prove that if we assume that, we reach a contradiction.

Denote by $Y_{I}^{\prime}=\sum_{i \in I} a_{i}^{(I)} Y_{i}$, and notice it depends on exactly the same set of variables from $Y_{1}, \ldots, Y_{k}$ as $Y_{I}$. By our assumption, there exist coefficients $\left\{\alpha_{i, I}\right\}$, not all zero, s.t. the polynomial

$$
h\left(Y_{1}, \ldots, Y_{k}\right)=\sum_{i \in[m], I \subseteq[k],|I| \leq \Delta\left(g_{i}\right)} \alpha_{i, I} g_{i}\left(x_{I}+Y_{I}^{\prime}\right)
$$

has bias at least $\rho$, where $\rho$ is a function of $\gamma, k$ and $m$ only (and not of $n$ ).

Fix $I_{0}$ maximal with regards to inclusion s.t. not all $\alpha_{i, I_{0}}$ are zero. Since we just care about the bias of $h$ under random $Y_{1}, \ldots, Y_{k}$, we can multiply each $Y_{i}$ by some non-zero coefficient. We thus assume w.l.o.g that $a_{i}^{\left(I_{0}\right)}=1$ for all $i \in I_{0}$. Let $\left|I_{0}\right|=r$. We assume w.l.o.g that $I_{0}=\{1,2, \ldots, r\}$. Notice that $Y_{[r]}^{\prime}=Y_{[r]}$. We also shorthand $x=x_{[r]}$.

Let $g_{i_{0}}$ be a polynomial with maximal degree $d^{\prime \prime} \leq d^{\prime}<d$ s.t. $\alpha_{i_{0}, I_{0}} \neq 0$.

We derive now once each of the variables in $Y_{1}, \ldots, Y_{r}$. Let $\left\{Z_{i}\right\}_{i=1 . . r}$ be new variables in $\mathbb{F}^{n}$, 
and consider:

$$
h^{\prime}\left(Y_{1}, \ldots, Y_{k}, Z_{1}, \ldots, Z_{r}\right)=\sum_{w \in\{0,1\}^{r}}(-1)^{|w|} h\left(Y_{1}+w_{1} Z_{1}, \ldots, Y_{r}+w_{r} Z_{r}, Y_{r+1}, \ldots, Y_{k}\right)
$$

First, by Lemma 9, $h^{\prime}$ has bias at least $\rho^{\prime}=\rho^{2^{k}}$.

Now, consider what happens to a term $g_{i}\left(x+Y_{I}^{\prime}\right)$ in $h$ after the derivation. If $I \neq[r]$, by the maximality of $I_{0}$ there must exist $i^{\prime} \in[r]$ s.t. $i^{\prime} \notin I$. Thus, deriving $Y_{i^{\prime}}$ zeroes out $g_{i}\left(x+Y_{I}^{\prime}\right)$.

So, the only terms remaining in $h^{\prime}$ come from terms in $h$ of the form $g_{i}\left(x+Y_{[r]}\right)$. Thus, $h^{\prime}$ does not depend on $Y_{i}$ for $i \notin[r]$, and also all the $g_{i}$ 's remaining must have $\Delta\left(g_{i}\right) \geq r$ (because $g_{i}\left(x+Y_{[r]}\right)$ appeared in $g$ with non-zero coefficient). Thus we can write:

$$
h^{\prime}=h^{\prime}\left(Y_{1}, \ldots, Y_{r}, Z_{1}, \ldots, Z_{r}\right)=\sum_{i \in[m]} \alpha_{i,[r]} \sum_{w \subseteq[r]}(-1)^{|w|} g_{i}\left(x+Y_{[r]}+Z_{w}\right)
$$

We now make an important observation. Notice that $h^{\prime}$ depends only on the sum $Y_{[r]}$, and not on the individual $Y_{1}, \ldots, Y_{r}$. So we can substitute $W=x+Y_{[r]}$ and get:

$$
h^{\prime}=h^{\prime}\left(W, Z_{1}, \ldots, Z_{r}\right)=\sum_{i \in[m]} \alpha_{i,[r]} \sum_{w \subseteq[r]}(-1)^{|w|} g_{i}\left(W+Z_{w}\right)
$$

We have assumed that $G$ is strong $\mathcal{F}$-regular. We will show now that if we choose $\mathcal{F}$ large enough, we have already reached a contradiction. Notice the polynomials $g_{i}\left(W+Z_{w}\right)$ are exactly those which appear in the regularity requirements ( where $X$ is replaced here by $W$, and $Y_{1}, Y_{2}, \ldots$ by $\left.Z_{1}, Z_{2}, \ldots\right)$. Let $G^{\prime}$ denote the set of $g_{i}$ 's s.t. $g_{i}$ appear in $h^{\prime}$ with non-zero coefficient.

We assume by induction that Theorem 2 holds for $d^{\prime \prime}<d$ and for all $n$. Since all polynomials $g_{i} \in G$ have degree at most $d-1$, then also $\operatorname{deg}\left(h^{\prime}\right) \leq d-1$, and so we can apply Theorem 2 on $h^{\prime}$. So, since $h^{\prime}$ has bias $\rho^{\prime}$, there must exist polynomials $q_{1}, \ldots, q_{t} \in \operatorname{Der}\left(h^{\prime}\right)$ s.t.

$$
h^{\prime}\left(W, Z_{1}, \ldots, Z_{r}\right)=Q\left(q_{1}\left(W, Z_{1}, \ldots, Z_{r}\right), \ldots, q_{t}\left(W, Z_{1}, \ldots, Z_{r}\right)\right)
$$

for some function $Q: \mathbb{F}^{t} \rightarrow \mathbb{F}$, s.t. $t=t\left(\rho^{\prime}, d^{\prime \prime}\right)$. Moreover, since every polynomial $q_{i}$ is of the form $h^{\prime}\left(W+a_{0}, Z_{1}+a_{1}, \ldots, Z_{r}+a_{r}\right)-h^{\prime}\left(W, Z_{1}, \ldots, Z_{r}\right)$ for some constants $a_{0}, \ldots, a_{r} \in \mathbb{F}^{n}$, and $h^{\prime}$ is the sum of $g_{i}\left(W+Z_{w}\right)$, we can decompose each $q_{i}$ to a sum of at most $2^{r}$ polynomials of the form $g_{i}\left(W+Z_{w}+a\right)-g_{i}\left(W+Z_{w}\right) \in \operatorname{Der}\left(G^{\prime}\right)$ for $w \subseteq\{0,1\}^{r}$. Let $q_{1}^{\prime}, \ldots, q_{t^{\prime}}^{\prime} \in \operatorname{Der}\left(G^{\prime}\right)$ denote these decomposed polynomials. We thus have that:

$$
h^{\prime}\left(W, Z_{1}, \ldots, Z_{r}\right)=Q^{\prime}\left(q_{1}^{\prime}\left(W+Z_{I_{1}^{\prime}}\right), \ldots, q_{t^{\prime}}^{\prime}\left(W+Z_{I_{t^{\prime}}^{\prime}}\right)\right)
$$

for some function $Q^{\prime}: \mathbb{F}^{t^{\prime}} \rightarrow \mathbb{F}, t^{\prime}=2^{r} t$ and $I_{1}^{\prime}, \ldots, I_{t}^{\prime} \subseteq[r]$. We got that we can compute

$$
h^{\prime}\left(W, Z_{1}, \ldots, Z_{r}\right)=\sum_{i \in[m]} \alpha_{i,[r]} \sum_{w \subseteq[r]}(-1)^{|w|} g_{i}\left(W+Z_{w}\right)
$$

as a function of $t^{\prime}$ polynomials of degree strictly smaller than $d^{\prime \prime}$. If we have $\mathcal{F}(m)>t^{\prime}$ this is a contradiction to the strong $\mathcal{F}$-regularity of $g_{1}, \ldots, g_{m}$.

Summarizing, there can be no linear combination of $\left\{g_{i}\left(x+Y_{I}\right): I \in S, 1 \leq|I| \leq \Delta\left(g_{i}\right)\right\}$ which has bias more than $\rho$, and so the distribution is $\gamma$-close to uniform. 


\section{From approximation to calculation: proof of Theorem 2}

In this section we prove Theorem 2, The main technical tool that we will use are Lemmas 6 and 7 , Let $p(X)$ stand for a degree $d$ polynomial with bias $\delta$. The proof of the theorem is immediate given the following two lemmas. The first lemma (Lemma 10) asserts that a biased degree $d$ polynomial can be approximated by constant many degree $d-1$ polynomials. This lemma was useful also in the proof of Green and Tao and its proof appears in [BV]. The second lemma (Lemma 11) asserts that approximation by few degree $d-1$ polynomials imply computation by few degree $d-1$ polynomials. In the following we present the two lemmas.

Lemma 10 (Bias imply approximation by few lower degree polynomials). Let $p(X)$ be a polynomial of degree $d$ with bias $\delta$. For any $\epsilon>0$ there exist polynomials $f_{1}(X), \ldots, f_{s}(X)$ of degree at most $d-1$ and a function $F: \mathbb{F}^{s} \rightarrow \mathbb{F}$ s.t.

$$
\mathbb{P}_{X \in \mathbb{F}^{n}}\left[F\left(f_{1}(X), \ldots, f_{s}(X)\right) \neq p(X)\right]<\epsilon
$$

The number $s$ of the polynomials depends only on $\delta$ and $\epsilon$. Moreover, $f_{1}, \ldots, f_{s} \in \operatorname{Der}(p)$.

The full proof can be found in [BV] (Lemma 24). The proof idea is that a random derivative in direction $a$ approximates $p(x)$ for any $x$, and so taking a majority value over enough random values of $a$ 's (but still a constant number) allows to compute $p$ on all but a $\epsilon$-fraction of the points.

Lemma 11 (Approximation by few lower degree polynomials imply computation by few lower degree polynomials). Let $p(X)$ be a polynomial of degree $d, f_{1}, \ldots, f_{s}$ polynomials of degree $d-1$, $(s=O(1))$ and $H: \mathbb{F}^{s} \rightarrow \mathbb{F}$ a function s.t. the composition $H\left(f_{1}(X), \ldots, f_{s}(X)\right) \epsilon_{d^{-}}$approximates $p$, where $\epsilon_{d}=2^{-\Omega(d)}$ Then there exist $s^{\prime}$ polynomials $f_{1}^{\prime}, \ldots, f_{s^{\prime}}^{\prime}$ and a function $H^{\prime}: \mathbb{F}^{s^{\prime}} \rightarrow \mathbb{F}$ s.t.

$$
H^{\prime}\left(f_{1}^{\prime}(X), \ldots, f_{s^{\prime}}^{\prime}(X)\right) \equiv p(X)
$$

Moreover, $s^{\prime}=s^{\prime}(d, s)$ (i.e. independent of $n$ ) and each $f_{i}^{\prime}$ if of the form $p(X+a)-p(X)$ or $f_{j}(X+a)$ for $a \in \mathbb{F}^{n}$.

Thus, to complete the proof of Theorem 2, it remains to prove Lemma 11

We start the proof of Lemma 11 by refining $F=\left\{f_{1}, \ldots, f_{s}\right\}$ to a strong-regular set. Let $\mathcal{F}$ be a large enough growth function (to be determined later). By Lemma 5 there exists a set $G=\left\{g_{1}, \ldots, g_{m}\right\}$ refining $F$, and a degree bound $\Delta$, s.t. $G$ is strong $\mathcal{F}$-regular with degree bound $\Delta$. Moreover, there exists a $C=C(\mathcal{F}, \delta, d)$ s.t. $G \subseteq \operatorname{Der}_{C}(F)$. We know that $G$ also approximates $p(X)$ at least as well as $F$ does. We will prove that it is in fact computes $F$ completely. We can then decompose each $g_{i} \in \operatorname{Der}_{C}(F)$ as a sum of at most $2^{C}$ elements in $\operatorname{Der}(p)$ to conclude the result.

Thus, we need to show that $G$ in fact computes $p(X)$ completely. For $c=\left(c_{1}, \ldots, c_{m}\right) \in \mathbb{F}^{m}$, denote by $R_{c} \subseteq \mathbb{F}^{n}$ the region

$$
R_{c}=\left\{x \in \mathbb{F}^{n}: g_{i}(x)=c_{i}\right\}
$$

To show that $G$ computes $p(X)$ is equivalent to showing that $p(X)$ is constant on any region $R_{c}$. Thus, we turn to study the regions $R_{c}$.

We first show (Lemma 12) that all regions $R_{c}$ have about the same volume, i.e. that they form an almost uniform division of $\mathbb{F}^{n}$ to $\mathbb{F}^{m}$ regions. Since $G$ is a strong regular refitment of $F$ that $\epsilon_{d}$-approximates $p$ we know that also $G \epsilon_{d}$-approximates $p$, i.e. there exists some $H^{\prime}: \mathbb{F}^{m} \rightarrow \mathbb{F}$ s.t.

$$
\mathbb{P}_{X \in \mathbb{F}^{n}}\left[H^{\prime}\left(g_{1}(X), \ldots, g_{m}(X)\right) \neq p(X)\right]<\epsilon_{d}
$$


For every region $R_{c}$, let $\eta_{c}$ be the probability that $p$ is different from $G$ on that region ( $G$ is constant on the region).

$$
\eta_{c}=\mathbb{P}_{X \in R_{c}}\left[p(X) \neq\left. G\right|_{R_{c}}\right]
$$

Since the average of $\eta_{c}$ is at most $\epsilon_{d}$, and all regions are almost uniform (Lemma 12) there can be at most $\sqrt{\epsilon_{d}}|\mathbb{F}|^{m}$ regions on which $\eta_{c}>\sqrt{\epsilon_{d}}$. We call these the bad regions, and we call the rest of the regions almost good regions. Next we show (Lemma 13) that the almost good regions are totally good and $p$ is fixed on them. Last, we use the fact that there are only few bad regions and $p$ is fixed on the rest to conclude that $p$ is also fixed on the bad regions (Lemma 15). Thus, $p(X)$ is in fact constant on all regions. To complete the proof of Lemma 11, it remains to prove Lemmas 12, 13 and 15.

Lemma 12 (Regions are uniform). Let $\gamma=\gamma(m)>0$ be a small enough error term. If $\mathcal{F}$ is large enough than

$$
\left|R_{c}\right|=|\mathbb{F}|^{n-m}(1 \pm \gamma)
$$

for all $c \in \mathbb{F}^{m}$.

Proof. Let $c \in \mathbb{F}^{m}$ and assume first that $R_{c}$ is not empty, i.e. there exist some $x$ s.t. $g_{i}(x)=c_{i}$ for all $i \in[m]$. We apply Corollary 8 with $k=1, x^{\prime}=x$ and $y_{1}=0$ and get:

$$
\mathbb{P}_{Y_{1}}\left[g_{i}\left(x+Y_{1}\right)=g_{i}(x), \forall i \in[m]\right]=|\mathbb{F}|^{-m}(1 \pm \gamma)
$$

Substituting $Y=x+Y_{1}$ proves the result for $R_{c}$.

To show the there can be no empty regions, assume otherwise. Thus, there are at most $|\mathbb{F}|^{m}-1$ non-empty cells, and each has volume at most $|\mathbb{F}|^{n-m}(1+\gamma)$. Thus $\left(|\mathbb{F}|^{m}-1\right)|\mathbb{F}|^{n-m}(1+\gamma) \geq|\mathbb{F}|^{n}$. If $\gamma(m)<|\mathbb{F}|^{-m}$ we get a contradiction. Thus, there are no empty regions, and so all regions have volume $|\mathbb{F}|^{n-m}(1 \pm \gamma)$.

Lemma 13 (Almost good regions are good). Let $R_{c}$ be a region s.t

$$
\mathbb{P}_{X \in R_{c}}[p(X)=b]>1-2^{-2(d+1)}
$$

for some constant $b \in \mathbb{F}$. Then $p(X)=b$ for all $X \in R_{c}$.

Before proving the lemma we need the following counting lemma on the number of hypercubes and pairs of hypercubes inside a region, similar to one in GT07. However, our technique avoids the need of integration.

Lemma 14. Let $\gamma=\gamma(m)>0$ be small enough error term, and assume $\mathcal{F}$ is large enough. For any point $R=R_{c}$ and a point $x \in R$ we have:

1. Let $Y_{1}, \ldots, Y_{d+1}$ be variables in $\mathbb{F}^{n}$. Then:

$$
\mathbb{P}_{Y_{1}, \ldots, Y_{d+1} \in \mathbb{F}^{n}}\left[x+Y_{I} \in R, \forall I \subseteq[d+1]\right]=|\mathbb{F}|^{-\sum_{i=1}^{m} \sum_{j=1}^{\Delta\left(g_{i}\right)}\left(\begin{array}{c}
d+1 \\
j
\end{array}\right)}(1 \pm \gamma)
$$

2. Let $Y_{1}, \ldots, Y_{d+1}, Z_{1}, \ldots, Z_{d+1}$ be variables in $\mathbb{F}^{n}$. For any non-empty $I_{0} \in[d+1]$ :

$$
\begin{aligned}
& \mathbb{P}_{Y_{1}, \ldots, Y_{d+1}, Z_{1}, \ldots, Z_{d+1} \in \mathbb{F}^{n}}\left[x+Y_{I} \in R, x+Z_{I} \in R, \forall I \subseteq[d+1] \mid Y_{I_{0}}=Z_{I_{0}}\right] \leq \\
& |\mathbb{F}|^{m}\left(|\mathbb{F}|^{-\sum_{i=1}^{m} \sum_{j=1}^{\Delta\left(g_{i}\right)}\left(\begin{array}{c}
d+1 \\
j
\end{array}\right)}\right)^{2}(1+\gamma)
\end{aligned}
$$


Proof. 1. This is a direct application of Corollary 8 for $k=d+1, x^{\prime}=x$ and $y_{1}, \ldots, y_{k}=0$.

2. Assume w.l.o.g that $I_{0}=\{1,2, \ldots, s\}$ for $1 \leq s \leq d+1$. We start by making a linear transformation on the coordinates to bring $Y_{I_{0}}$ and $Z_{I_{0}}$ to a single variable. Let $Y_{i}^{\prime}=Y_{i}$ for $i \neq s$ and $Y_{s}^{\prime}=Y_{1}+\ldots+Y_{s}$, and similarly define $Z_{1}^{\prime}, \ldots, Z_{d+1}^{\prime}$. We write $Y_{I}$ in the basis of $Y_{1}^{\prime}, \ldots, Y_{d+1}^{\prime}$. Divide $I=I_{s} \cup I_{\bar{s}}$ where $I_{s}=I \cap[s]$ and $I_{\bar{s}}=I \backslash I_{s}$. We have:

- If $s \notin I, Y_{I}=\sum_{i \in I} Y_{i}^{\prime}$

- If $s \in I, Y_{I}=Y_{s}^{\prime}-\sum_{i \in[s] \backslash I_{s}} Y_{i}^{\prime}+\sum_{i \in I_{\bar{s}}} Y_{i}^{\prime}$

Consider for every $I$ the set $T_{I}$ of indices of $Y_{i}^{\prime}$ which appear in the expansion of $Y_{I}$. Notice that for any $T \subseteq[d+1]$ there is exactly one $I$ s.t. $T_{I}=T$. In particular, in order that $g_{i}\left(x+Y_{I}\right)=g_{i}(x)$ for all $I$, we must have in particular that:

- For any $I \subseteq[d+1]$ s.t. $s \notin I$ and $|I| \leq \Delta\left(g_{i}\right)$,

$$
g_{i}\left(x+Y_{I}^{\prime}\right)=g_{i}(x)
$$

- For any $I \subseteq[d+1]$ s.t. $s \in I$ and $|I| \leq \Delta\left(g_{i}\right)$,

$$
g_{i}\left(x+Y_{s}^{\prime}-Y_{I \cap[s-1]}^{\prime}+Y_{I \cap\{s+1, \ldots, d+1\}}^{\prime}\right)=g_{i}(x)
$$

Similarly for the $Z^{\prime}$ 's, using the fact that the event $Y_{I_{0}}=Z_{I_{0}}$ translates to $Z_{s}^{\prime}=Y_{s}^{\prime}$ :

- For any $I \subseteq[d+1]$ s.t. $s \notin I$ and $|I| \leq \Delta\left(g_{i}\right)$,

$$
g_{i}\left(x+Z_{I}^{\prime}\right)=g_{i}(x)
$$

- For any $I \subseteq[d+1]$ s.t. $s \in I$ and $|I| \leq \Delta\left(g_{i}\right)$,

$$
g_{i}\left(x+Y_{s}^{\prime}-Z_{I \cap[s-1]}^{\prime}+Z_{I \cap\{s+1, \ldots, d+1\}}^{\prime}\right)=g_{i}(x)
$$

The probability of this event is an upper bound on our required probability. Since our variables

$$
Y_{1}^{\prime}, \ldots, Y_{d+1}^{\prime}, Z_{1}^{\prime}, . ., Z_{s-1}^{\prime}, Z_{s+1}^{\prime}, \ldots, Z_{d+1}^{\prime}
$$

are uniform and independent, we can apply Lemma 7 to show that its probability is the required upper bound. The number of subsets of size $j>1$ in the above events is $\left(\begin{array}{c}d+1 \\ j\end{array}\right)$ for the event on the $Y^{\prime}$ s, and also $\left(\begin{array}{c}d+1 \\ j\end{array}\right)$ for the event on $Z_{1}^{\prime}, \ldots, Z_{s-1}^{\prime}, Y_{s}^{\prime}, Z_{s+1}^{\prime}, \ldots, Z_{d+1}^{\prime}$. For $j=1$ however we have intersection ( $Y_{s}^{\prime}$ is appearing twice), and so the number of events is $2\left(\begin{array}{c}d+1 \\ 1\end{array}\right)-1$. Thus, by Lemma 7 the probability of the total event is:

$$
|\mathbb{F}|^{m}\left(|\mathbb{F}|^{-\sum_{i=1}^{m} \sum_{j=1}^{\Delta\left(g_{i}\right)}\left(\begin{array}{c}
d+1 \\
j
\end{array}\right)}\right)^{2}(1 \pm \gamma)
$$

which upper bounds the required probability.

We now prove Lemma 13 using Lemma 14. We follow the same proof as in GT07. 
Proof. Let $B \subseteq R$ be the set of all "bad" points $x \in R$ on which $p(x) \neq b$. By our assumption, $|B|<2^{-2(d+1)}|R|$. Assume $B$ is non-empty, and choose some $x \in B$. Let $Y_{1}, \ldots, Y_{d+1}$ be random variables in $\mathbb{F}^{n}$. Fix small enough $\gamma=\gamma(m)$. By Lemma 14 (1),

$$
p_{R}=\mathbb{P}\left[x+Y_{I} \in R, \forall I \subseteq[d+1]\right] \geq|\mathbb{F}|^{-\sum_{i=1}^{m} \sum_{j=1}^{\Delta\left(g_{i}\right)}\left(\begin{array}{c}
d+1 \\
j
\end{array}\right)}(1-\gamma)
$$

We now wish to bound the event that when all $X+Y_{I}$ are in $R$, some $X+Y_{I}$ is in $B$, and then union bound over all possible $I$.

We start by applying Cauchy-Schwartz to transform the problem to counting pairs of hypercubes. Fix some non-empty $I_{0} \subseteq[d+1]$, and let

$$
\begin{aligned}
p_{B}= & \mathbb{P}\left[x+Y_{I} \in R \forall I \subseteq[d+1] \wedge x+Y_{I_{0}} \in B\right]= \\
& \sum_{x_{0} \in B} \mathbb{P}\left[x+Y_{I} \in R \forall I \subseteq[d+1] \wedge x+Y_{I_{0}}=x_{0}\right]
\end{aligned}
$$

We need to upper bound $p_{B}$.

$$
\begin{aligned}
p_{B}^{2}= & \left(\sum_{x_{0} \in B} \mathbb{P}\left[x+Y_{I} \in R \forall I \subseteq[d+1] \wedge x+Y_{I_{0}}=x_{0}\right]\right)^{2} \leq \\
& |B| \sum_{x_{0} \in B} \mathbb{P}\left[x+Y_{I} \in R \forall I \subseteq[d+1] \wedge x+Y_{I_{0}}=x_{0}\right]^{2}= \\
& |B| \mathbb{P}\left[x+Y_{I} \in R \forall I \subseteq[d+1] \wedge x+Z_{I} \in R \forall I \subseteq[d+1] \wedge x+Y_{I_{0}}=x+Z_{I_{0}}\right]= \\
& |B||\mathbb{F}|^{-n} \mathbb{P}\left[x+Y_{I} \in R, x+Z_{I} \in R \forall I \subseteq[d+1] \mid x+Y_{I_{0}}=x+Z_{I_{0}}\right]
\end{aligned}
$$

where $Z_{1}, \ldots, Z_{d+1}$ are new variables in $\mathbb{F}^{n}$.

By claim (2) in Lemma 14 we get that this probability is at most

$$
|B||\mathbb{F}|^{m-n} p_{R}^{2}(1+\gamma)
$$

By Lemma 12, $|R|=|\mathbb{F}|^{n} \mathbb{P}_{X \in \mathbb{F}^{n}}[X \in R]=|\mathbb{F}|^{n-m}(1 \pm \gamma)$. Thus, we have that:

$$
p_{B}^{2} \leq \frac{|B|}{|R|} p_{R}^{2}(1 \pm 2 \gamma) \leq 2^{-2(d+1)} p_{R}^{2}
$$

and thus $\frac{p_{B}}{p_{R}} \leq 2^{-(d+1)}(1 \pm 2 \gamma)$. We can now union bound over all non-empty $I_{0} \subseteq[d+1]$. The probability that there is some $I_{0}$ for which $x+Y_{I_{0}} \in B$ is at most

$$
\left(2^{d+1}-1\right)\left(2^{-(d+1)}+\gamma\right)<1
$$

for small enough $\gamma$.

Thus, there must exist $y_{1}, \ldots, y_{d+1} \in \mathbb{F}^{n}$ s.t.

$$
x+y_{I} \in R \backslash B
$$


for all non-empty $I \subseteq[d+1]$. Equivalently, $p\left(x+y_{I}\right)=b$ for all such $I$ 's. However, since $p(X)_{y_{1}, \ldots, y_{d+1}} \equiv 0$,

$$
p(x)=\sum_{I \subseteq[d+1],|I|>0}(-1)^{|I|+1} p\left(x+y_{I}\right)
$$

and so if all $p\left(x+y_{I}\right)=b$, then also $p(x)=b$, hence $x \notin B$. So we have proved that $B$ is empty, i.e. $p$ is constant on $R$.

We finish the proof of Theorem 2 by proving that if $p(X)$ is constant over almost all regions, then it must be constant over any region.

Lemma 15 (If almost all regions are totally good, all are totally good). Assume that the fraction of regions on which $p$ is constant is at least $1-2^{-(d+2)}$. Then $p$ is constant over any region.

Proof. Let $R$ be any region, and $x, x^{\prime} \in R$ two points in $R$. We need to show that $p(x)=p\left(x^{\prime}\right)$. Choose $y_{1}^{\prime}, \ldots, y_{d+1}^{\prime} \in \mathbb{F}^{n}$ randomly. The probability that $x^{\prime}+y_{I}^{\prime}$ falls in a bad region for any nonempty $I \subseteq[d+1]$ is $2^{-(d+2)}$ (since regions are almost uniform, see Lemma 12). Thus, applying union bound over all non-empty $I \subseteq[d+1]$ we get that $\left\{x^{\prime}+y_{I}^{\prime}\right\}$ fall in good regions for all non-empty $I$ with probability at least $1 / 2$. Fix some $y_{1}^{\prime}, \ldots, y_{d+1}^{\prime}$ fulfilling this requirement.

Let $Y_{1}, \ldots, Y_{d+1} \in \mathbb{F}^{n}$ be random variables. Since $g_{i}(x)=g_{i}\left(x^{\prime}\right)$ for all $i \in[m]$ we can apply Corollary 8 :

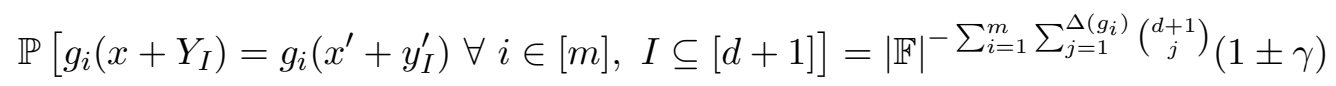

In particular, for small enough $\gamma$ we get that

$$
\mathbb{P}\left[g_{i}\left(x+Y_{I}\right)=g_{i}\left(x^{\prime}+y_{I}^{\prime}\right) \forall i \in[m], I \subseteq[d+1]\right]>0
$$

Let $y_{1}, \ldots, y_{d+1}$ be such assignment to $Y_{1}, \ldots, Y_{d+1}$. We thus have that for all non-empty $I \subseteq[d+1]$ and for all $i \in[m], g_{i}\left(x+y_{I}\right)=g_{i}\left(x^{\prime}+y_{I}^{\prime}\right)$. Since the region of $x^{\prime}+y_{I}^{\prime}$ is good for all non-empty $I$, we get that for all non-empty $I \subseteq[d+1]$,

$$
p\left(x+y_{I}\right)=p\left(x^{\prime}+y_{I}^{\prime}\right)
$$

We now use the fact that $p$ is a degree $\mathrm{d}$ polynomial. If we derive $p d+1$-times in any direction, we will always get zero. We thus have that for $x, y_{1}, \ldots, y_{d+1} \in \mathbb{F}^{n}$ :

$$
\sum_{I \subseteq[d+1]}(-1)^{|I|} p\left(x+y_{I}\right)=0
$$

Since the same identity is true for $x^{\prime}, y_{1}^{\prime}, \ldots, y_{d+1}^{\prime}$, we get that $p(x)=p\left(x^{\prime}\right)$.

Acknowledgement We would like to thank Avi Wigderson, Noga Alon and Terrence Tao for helpful discussions. The second author would like to thank his advisor, Omer Reingold, for his help and support. 


\section{References}

[GT07] B. Green, T.Tao, The distribution of polynomials over finite fields, with applications to the Gowers norms, preprint, 2007.

[BV] A. Bogdanov and E. Viola. Pseudorandom bits for polynomials via the Gowers norm. In the 48th Annual Symposium on Foundations of Computer Science (FOCS 2007).

[MS] J. MacWilliams and N. J. A. Sloane, The Theory of Error Correcting Codes, Amsterdam, North-Holland, 1977.

[LMS] S. Lovett, R. Meshulam and A. Samorodnitsky, The Inverse Conjecture for the Gowers Norm is False, to appear in the 40th ACM Symposium on Theory of Computing (STOC 2008).

[DLMORSW] Ilias Diakonikolas, Homin K. Lee, Kevin Matulef, Krzysztof Onak, Ronitt Rubinfeld, Rocco A. Servedio, Andrew Wan Testing for Concise Representations. the 48th Annual Symposium on Foundations of Computer Science (FOCS 2007).

[RS] , Ronitt Rubinfeld and Madhu Sudan, Robust characterizations of polynomials with applications to program testing, SIAM Journal on Computing, 25 (2), 252-271, 1996. 